\title{
MANY-ELECTRON EFFECTS IN LOW-DIMENSIONAL SEMICONDUCTOR STRUCTURES
}

\author{
P. HAWRYLAK \\ Institute for Microstructural Sciences, National Research Council of Canada \\ Ottawa, K1A OR6, Canada
}

\begin{abstract}
Many-electron effects in low-dimensional semiconductor structures: quantum wells, wires, and dots are discussed. Three examples are given: (a) the quasi-particles of a Fermi liquid are illustrated with the calculation of the self-energy of a hot electron coupled to plasmons and optical phonons in modulation doped quantum wells, (b) the Fermi edge singularity and (c) the incompressible states of strongly correlated electrons in quantum dots tunable by applied magnetic field.
\end{abstract}

PACS numbers: $73.20 . \mathrm{Dx}$

\section{Introduction}

There is a number of phenomena in the physics of low-dimensional semiconductor structures which cannot be understood in the single-particle picture but require a treatment of either the collective behavior of all electrons, correlations built into electronic states due to Pauli exclusion principle, or both. The collective excitation spectrum (plasmons) [1], the Fermi edge singularity (FES) [2], and incompressible states of correlated electrons in quantum dots [3] illustrate nicely these three cases.

\section{Collective and single-particle properties of modulation doped quantum wells}

In quantum wells, superlattices, and heterojunctions many electrons "communicate with each" other via long ranged Coulomb interactions. The effect of reduced dimensionality manifests itself in the collective excitation spectrum which depends on geometry of the structure [1]. The collective excitation spectrum is measured directly via resonant electronic Raman scattering [4]. It determines such properties as hot electron scattering rate, mean free path, and effective mass [5]. These quantities can be calculated from electron's self-energy within the Fermi 
liquid approach. We will discuss a realistic calculation of the self-energy of a hot electron coupled to plasmons and optical phonons in a modulation doped quantum well [6].

Let us consider a degenerate electron gas with density $n_{\mathrm{s}}$ and Fermi energy $E_{\mathrm{F}}$ populating the lowest subband of a quantum well of width $a$. Because of the scattering by the excitations of the electron gas and by optical phonons, electron energy $E_{k}$ is changed to $E_{k}+\Sigma_{k}$. The scattering rate $1 / \tau$ is simply given by the imaginary part of the self-energy $\operatorname{Im}(\Sigma): 1 / \tau=(2 / \hbar) \operatorname{Im} \Sigma\left(k, \xi_{k}^{\mathrm{e}}\right)$, where $\xi_{k}^{\mathrm{e}}$ is the kinetic energy measured with respect to the Fermi level $\mu, \xi_{k}^{\mathrm{e}}=\hbar^{2} k^{2} / 2 m_{\mathrm{e}}-\mu_{\mathrm{e}}$. The self-energy can be calculated diagrammatically [5] as shown in Fig. 1a, and written schematically as $\Sigma=W^{0} \Gamma G^{0}$. Here $\Gamma$ is the vertex correction, $W^{0}$ is screened
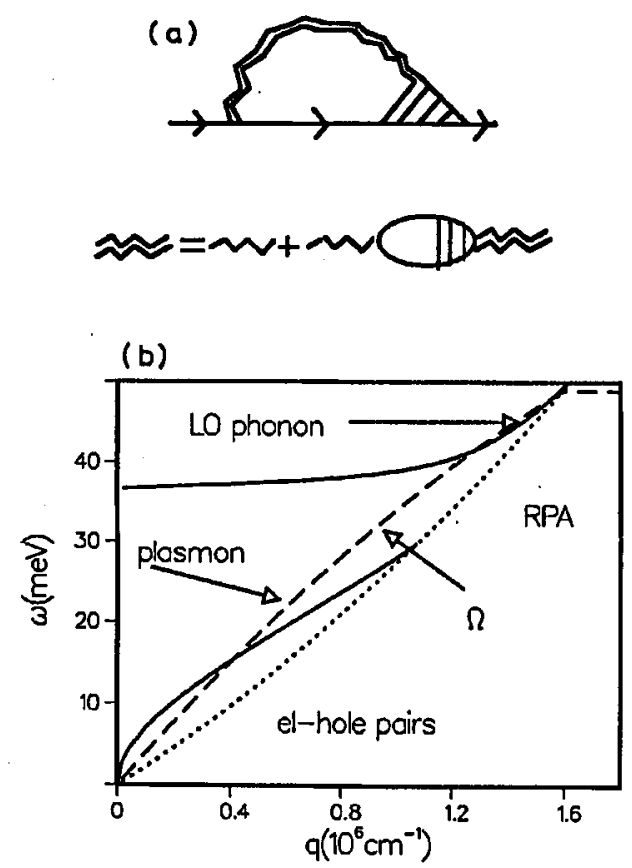

Fig. 1. (a) Diagrammatic representation of self-energy $\Sigma(k, \omega)$, effective interaction $W(q, \omega)$, and vertex function, (b) the excitation spectrum: solid lines give collective modes (plasmon-like and LO-phonon like), dashed line gives the onset of electron-hole continuum, and broken line shows the kinematic restriction $\Omega_{+}(q)$ for an electron with excess kinetic energy of $\approx 50 \mathrm{meV}$; modulation doped quantum well with carrier density $n=6 \times 10^{11} \mathrm{~cm}^{-2}$, well width $a=60 \AA$, and Fermi energy $E_{\mathrm{F}}=21 \mathrm{meV}$. The GaAs effective electron mass of 0.068 has been used.

interaction which depends on $\Gamma$, and $G^{0}$ is the noninteracting Green function. Three different methods of calculating the scattering time: random phase approximation (RPA) [5], Hubbard (H) [7], and Vignale, $\mathrm{Ng}$, and Singwi (VNS) [8] are used. These three approximations to the spectral function $W_{\mathrm{ee}}=W^{0}(\Gamma) \Gamma$ of the effective interaction can be written simply in terms of the Lindhard polarizability 
$\Pi^{0}$ of the 2D electron gas, the bare interaction $W^{0}$, and static local fields $G(q)$. For example, the Hubbard approximation for the self-energy can be written as $\Sigma=W^{0} \Gamma_{\mathrm{H}} /\left(1-W^{0} \Pi^{0} \Gamma_{\mathrm{H}}\right)$ where the Hubbard vertex correction is given in terms of the local field $G(q)$ as $\Gamma_{\mathrm{H}}=1 /\left(1+W^{0} \Pi^{0} G\right)$. We can combine our approximations for the effective interaction $W_{\mathrm{ee}}$ which incorporates vertex corrections in a very simple way $[6]$ :

$$
\begin{array}{ll}
\text { RPA } & \operatorname{Im}\left[W_{\text {ee }}(q, \omega)\right]=\operatorname{Im}\left[W^{0} /\left(1-W^{0} \Pi^{0}\right)\right] \\
\text { Hubbard } & \operatorname{Im}\left[W_{\text {ee }}(q, \omega)\right]=\operatorname{Im}\left\{W^{0} /\left[1-W^{0}(1-G) \Pi^{0}\right]\right\} \\
\text { VNS } & \operatorname{Im}\left[W_{\text {ee }}(q, \omega)\right]=\operatorname{Im}\left\{W^{0}(1-G) /\left[1-W^{0}(1-G) \Pi^{0}\right]\right\}
\end{array}
$$

The last two approximations attempt to incorporate vertex corrections into the calculation of self-energy. In the Hubbard approximation the effective interaction $W^{0}(1-G)$ is modified by local fields $G$ only in the effective dielectric function, i.e. in the screening cloud. This renormalizes the frequency spectrum of collective modes. It does not affect the coupling strength of the photo-excited electron with renormalized excitations. Due to a cancellation between vertex corrections in the coupling of the hot electron to the surrounding it electrons and vertex corrections in the screening cloud, the net result in Hubbard approximation is quite similar to the RPA result which neglects vertex corrections altogether. However, in the VNS approximation the local fields affect both the interaction within the screening cloud and the coupling of the hot electron with excitations of the electron gas.

In all three cases the spectral function gives nonvanishing contributions when either $\operatorname{Im}\left(\Pi^{0}\right)$ is nonzero (single particle excitations) or the denominator vanishes (for $\operatorname{Im}\left(\Pi^{0}\right)=0$ ) (collective excitations). The spectrum of collective excitations is associated with zeros of the dielectric function. The local fields are approximated by $G(q)=q / 2\left(q^{2}+k_{\mathrm{F}}^{2}\right)^{1 / 2}$. The bare effective electron-electron interaction $W^{0}$ is a sum of the bare Coulomb interaction and the electron-phonon interaction. The interaction with optical bulk, interface, and confined phonons is a nontrivial issue. However, the energy dependence of the scattering rate of a single electron due to interactions with all possible phonon modes of a quantum well turns out to be well approximated by the effective bulk phonon model [9], adopted here for simplicity. The bare effective electron-electron interaction is now simply a high frequency electron-electron interaction matrix element $W_{\infty}=2 \pi e^{2} F(q) / \varepsilon_{\infty} q(F(q)$ is a form factor and $\varepsilon_{\infty}$ is the high frequency dielectric constant) screened by the frequency dependent dielectric constant $\varepsilon(\omega)=\left(\omega^{2}-\omega_{\text {LO }}^{2}\right) /\left(\omega^{2}-\omega_{\text {TO }}^{2}\right)$, i.e. $W^{0}=W_{\infty} / \varepsilon(\omega)$.

We can now write a simple expression for the scattering rate of a hot electron. The imaginary part of the electron self-energy in 2D at zero temperature can be written as a product of the occupation of final states, the spectral function of effective interaction, and a kinematic factor $[5,6]$ :

$$
\operatorname{Im} \Sigma_{\mathrm{e}}\left(k, \xi_{k}^{\mathrm{e}}\right)=\int_{0}^{\infty} \frac{\mathrm{d} q q}{2 \pi} \int_{0}^{\Omega_{+}(k, q)} \frac{\mathrm{d} \omega}{2 \pi} \frac{\left[1-f\left(\xi_{k}^{\mathrm{e}}-\omega\right)\right]\left[-2 \operatorname{Im} W_{\mathrm{ee}}(q, \omega)\right]}{2 \pi \sqrt{\left[\omega-\Omega_{-}(k, q)\right]\left[\Omega_{+}(k, q)-\omega\right]}} .
$$

Here $f(E)$ is a Fermi function, $W_{\mathrm{ee}}$ is the effective interaction, and frequencies $\Omega_{+(-)}$, given by $\Omega_{+(-)}=\xi_{k}-\xi_{k-(+) g}$, correspond simply to the energy of the excitation generated in forward $(-)$ and backward $(+)$ scattering of a hot electron. The self-energy is given by the sum of all possible scattering processes of an electron 
with energy $\xi_{k}$ into empty states (factor $(1-f)$ ) with the emission of an excitation with frequency $\omega$ and wave vector $q$. The spectrum of excitations is given by the spectral function $\operatorname{Im}\left(W_{\mathrm{ee}}(q, \omega)\right)$ of the effective interaction. The frequency and wave vector integral is weighted by a kinematic factor which reflects the number of final states available in the scattering process. The kinematic factor and hence the frequency integral diverges at frequency $\Omega_{+}$. Excitations with frequency $\omega$ close to $\Omega_{+}$give major contribution to the scattering time.

In Fig. 1b we show a typical RPA excitation spectrum of the coupled electronphonon system for a quantum well of width $a=60 \AA$, carrier density $n_{\mathrm{s}}=$ $6 \times 10^{11} \mathrm{~cm}^{-2}$, and electron Fermi energy of $21 \mathrm{meV}$. The solid lines show coupled collective modes: the shifted plasmon-like (lower mode) and LO phonon-like collective mode (upper mode). Collective modes merge into the continuum of electron-hole pair excitations of the electron gas, denoted by a dashed line. The broken line shows the backscattering frequency $\Omega_{+}$for an electron with an excess kinetic energy of $\approx 50 \mathrm{meV}$. Only excitations with frequency less than $\Omega_{+}$are allowed, and both phonon-like and plasmon-like excitations contribute to the scattering rate. Qualitatively similar excitation spectra are obtained for the Hubbard and VNS approximations but with slightly lower frequencies.

Given the excitation spectrum we now calculate dephasing times using Eqs. (1), (2). The scattering rate for a quasiparticle is shown in Fig. 2a for a modulation doped quantum well with $n=6 \times 10^{11} \mathrm{~cm}^{-2}, a=60 \AA$, effective mass $m=0.068$, and $E_{\mathrm{F}}=21 \mathrm{meV}$. The low energy contribution $(E<30 \mathrm{meV})$ corresponds to the scattering by single-particle excitations. The rapid rise in scattering rate around $30 \mathrm{meV}$ is associated with the onset of plasmon emission. The step like rise in the scattering rate for excess kinetic energy of approximately $50 \mathrm{meV}$ is associated with the emission of the $\omega_{\mathrm{LO}}$-like mode. The calculated results compare well with scattering times measured in four wave mixing (FWM) experiments (filled squares) by Kim et al. [10], but more experiments are needed to identify the plasmon scattering threshold. This can be accomplished in lower density samples for which the coupling of optical phonons and plasmons is reduced and the two thresholds in scattering rate are well separated. We show in Fig. $2 b$ the RPA, Hubbard, and VNS approximations to the electron scattering rate for a lower density sample, i.e. modulation doped quantum well with $n=2 \times 10^{11} \mathrm{~cm}^{-2}, a=80 \AA$, and $E_{\mathrm{F}}=7 \mathrm{meV}$. The striking feature in the lower density samples is the shift of the threshold due plasmon emission to much lower excess quasiparticle energy. The RPA calculation gives a larger scattering rate than either the Hubbard or the VNS approximation.

To conclude, charge oscillations in the electron liquid couple to optical phonons and lead to collective excitations which are dimensionality dependent. The screening process depends on exchange-correlation hole surrounding individual electrons viewed here as Landau quasiparticles. These excitations scatter not bare electrons but dressed quasiparticles. The sum of all scattering processes in modulation doped quantum wells is quenched close to the Fermi edge due to phase space blocking but strongly enhanced away from the Fermi edge due to plasmon emission. The enhancement provides a useful spectroscopic tool with which to study short range correlations in low-dimensional semiconductor structures. 


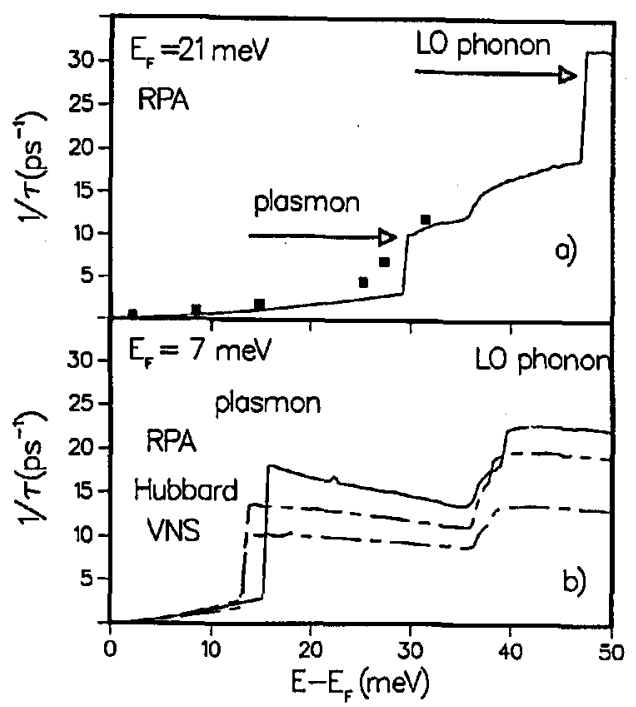

Fig. 2. (a) The dependence of the scattering rate $1 / \tau$ on excess kinetic energy $E$ of the hot electron measured with respect to Fermi energy. $E_{F}$ for a modulation doped quantum well with carrier density $n=6 \times 10^{11} \mathrm{~cm}^{-2}$, well width $a=60 \AA$, and Fermi energy $E_{\mathrm{F}}=21 \mathrm{meV}$. (b) The dependence of $1 / \tau$ on excess kinetic energy for a modulation doped quantum well with carrier density $n=2 \times 10^{11} \mathrm{~cm}^{-2}$, well width $a=80 \AA$, and Fermi energy $E_{\mathrm{F}}=7 \mathrm{meV}$. The solid line corresponds to the RPA result, the upper dashed line gives Hubbard approximation, and the lower dashed line the VNS approximation.

\section{Fermi edge singularity}

Excitonic effects in optical properties of semiconductors correspond to the correlation of a photo-excited electron in the conduction band with a photo-excited hole in the valence band. This attractive interaction leads to bound exciton states which manifests themselves as sharp peaks in the single-particle energy gap. In doped samples holes are correlated not only with the photo-excited electron but also with all electrons present in the conduction band. No excitons exist but many-electron hole correlations lead to sharp features in absorption and emission spectra in the vicinity of the Fermi level, the Fermi edge singularity [2], even in highly doped samples. The explanation of these effects requires a proper treatment of dynamical self-energy and vertex corrections especially for low-dimensional systems (quantum wells and wires) where exciton binding energy is typically comparable to the Fermi energy. The origin of the Fermi edge singularity is associated with the peculiar overlap of many-electron initial and final state wave functions $[2,11]$, i.e. with Pauli exclusion principle.

\section{Correlated electrons in quantum dots}

In this example we will discuss strongly correlated and interacting elec- 
trons in quantum dots. Quantum dots are zero-dimensional structures containing a small and controlled number of electrons [3]. Because electrons cannot effectively avoid each other the physics of quantum dots can only be understood when electron-electron interactions and electron correlations are taken into account. This can be accomplished by employing exact numerical diagonalization techniques and exploiting single- and many-particle symmetries. The ground state of a quantum dot in a magnetic field depends on the competition of kinetic, Zeeman, and electron-electron interaction energies [12-14]. This competition leads to a series of incompressible states with "magic angular momentum" values. One can think of these states as "precursors" of Laughlin states responsible for the fractional quantum Hall effect [15]. We will also describe how these incompressible states can be observed in far infrared (FIR) [16], photoluminescence (PL) [14], Raman, and single electron capacitance (SECS) spectroscopies [12, 17].

\subsection{The model}

Let us consider a two-dimensional artificial atom (dot) containing $N$ electrons confined by an externally imposed parabolic potential with a characteristic energy $\omega_{0}$ and moving in the field of a fictitious nucleus (gate) with a positive charge $+\mathrm{Ne}$ at a distance $d$ away from the plane of the dot [13]. The atom is placed in a magnetic field $B$ normal to the plane of the dot. The positive charge assures charge neutrality of the atom and plays the role of the gate. For a dot size much smaller than $d$, we can approximate the nonsingular potential $V(r)$ of the positive charge by $-e^{2} N / \varepsilon_{0} d+\frac{1}{2}\left(e^{2} N / \varepsilon_{0} d^{3}\right) r^{2}$. Therefore the positive charge contributes a constant term and a parabolic term. The larger the number of carriers $N$, the stronger the single particle confinement. The Hamiltonian for $N$ electrons can be written as

$$
\begin{aligned}
H= & \sum_{i=1}^{N}\left[-\alpha N+\frac{1}{2 m}\left(p_{i}+\frac{e}{c} A_{i}\right)^{2}+\frac{1}{2} m \omega_{N}^{2} r_{i}^{2}+g \mu_{\mathrm{B}} S_{z}^{i} B\right] \\
& +\sum_{\substack{i<j \\
i, j=1}}^{N} \frac{e^{2}}{\varepsilon_{0}\left|r_{i}-r_{j}\right|} .
\end{aligned}
$$

The vector potential is given in the symmetrical gauge, $g$ is the effective $g$-factor, $\mu_{\mathrm{B}}$ is the Bohr magneton, $S_{z}^{i}$ is the $z$-component of the $i$-th particle spin, and $\alpha=e^{2} / \varepsilon_{0} d$. The effective confining frequency $\omega_{N}$ depends on the number of particles and is given by $\omega_{N}=\left[\left(\omega_{0}\right)^{2}+\left(e^{2} N / m \varepsilon_{0} d^{3}\right)\right]^{1 / 2}$, and we take $\hbar=1$.

To diagonalize the single particle Hamiltonian of the dot one defines a pair of harmonic oscillator lowering (raising) operators $(a, b)$ for each particle [13, 14]:

$$
\begin{aligned}
z^{*} & =\sqrt{l_{0}^{2} / 2 \Omega}\left(a^{+}+b\right), & z & =\sqrt{l_{0}^{2} / 2 \Omega}\left(a+b^{+}\right), \\
\partial_{z} & =\sqrt{\Omega / 2 l_{0}^{2}}\left(b-a^{+}\right), & \partial_{z}^{*} & =\sqrt{\Omega / 2 l_{0}^{2}}\left(a-b^{+}\right),
\end{aligned}
$$

where $z=(x-\mathrm{i} y) / 2, l_{0}=l /\left(m \omega_{\mathrm{c}}\right)^{1 / 2}$ is the magnetic length, $\omega_{\mathrm{c}}$ is the cyclotron energy and $\Omega=\left(1+\left(2 \omega_{N} / \omega_{c}\right)^{2}\right)^{1 / 2}$. The single-particle Hamiltonian for an electron in the dot reduces to the Hamiltonian of a two-dimensional anisotropic harmonic 
oscillator with eigenenergies $E_{n, m}=\Omega_{+}(n+1 / 2)+\Omega_{-}(m+1 / 2)$, eigenstates $|m, n\rangle$ given by $|m, n\rangle=\left(b^{+}\right)^{m}\left(a^{+}\right)^{n}|0\rangle /(n ! m !)^{1 / 2}$ and two frequencies $\Omega_{+(-)}=$ $\left[\sqrt{\omega_{\mathrm{c}}^{2}+4 \omega_{N}^{2}}+(-) \omega_{\mathrm{c}}\right] / 2$.

The explicit separation of the CM (center of mass) and the relative motion of electrons is achieved through a generalized Jacobi transformation [13] $U_{l m}=\exp [\mathrm{i}(l-1) m 2 \pi / N] / \sqrt{N}$, transforming individual particle boson creation operators $\left(a_{m}^{+}\right)$into $\mathrm{CM}$ and relative creation operators $\left(A_{m}^{+}\right): A_{l}^{+}=\sum_{m} U_{l m} a_{m}^{+}$. It is easy to see that the transformation $U$ preserves boson commutation relations among operators $(A, B)$ and that the center of mass motion separates from the Hamiltonian. The CM Hamiltonian is simply given by $H_{\mathrm{CM}}=\Omega_{+}\left(A_{1}^{+} A_{1}+1 / 2\right)+$ $\Omega_{-}\left(B_{1}^{+} B_{1}+1 / 2\right)$, and index 1 stands for a CM particle. The motion of $(N-1)$ relative particles is determined by electron-electron interactions and governed by the relative Hamiltonian

$$
\begin{aligned}
H_{\text {rel }} & =\sum_{m=2}^{N} \Omega_{+}\left(A_{m}^{+} A_{m}+1 / 2\right)+\Omega_{-}\left(B_{m}^{+} B_{m}+1 / 2\right) \\
& +\sum_{\boldsymbol{q}, i<j}^{N} v(q) \mathrm{e}^{-2|Q|^{2}} \exp \left(\mathrm{i} \sum_{m=2}^{N} Q_{i j m}^{+} A_{m}^{+}\right) \exp \left(\mathrm{i} \sum_{m=2}^{N} Q_{i j m} A_{m}\right) \\
& \times \exp \left(\mathrm{i} \sum_{m=2}^{N} Q_{i j m} B_{m}^{+}\right) \exp \left(\mathrm{i} \sum_{m=2}^{N} Q_{i j m}^{+} B_{m}\right),
\end{aligned}
$$

where $Q_{i j m}^{+}=Q^{*}\left(U_{i m}^{+}-U_{j m}^{+}\right), Q=\left(q_{x}+q_{y}\right) l_{0} / \sqrt{2 \Omega}$ and $v(q)=2 \pi e^{2} / \varepsilon_{0} q$. The relative Hamiltonian depends only on relative operators $A_{m}$, and the FIR spectroscopy [16] measures the bare confining potentials, i.e. frequencies $\Omega_{(+,-)}$.

The states of the relative Hamiltonian are built from simple products of single particle states $\prod_{i=2}^{N}\left|N_{i} M_{i}\right\rangle$ and spin states by the use of the antisymmetric operator $\hat{A} s=\sum_{i=1}^{N !} \operatorname{Det}\left(P_{i}\right) P_{i}$, where $P_{i}$ are elements of the $(N-1)$-dimensional representation of the permutation group $S_{N}$. The construction of the $(N-1)$ representations of the $S_{N}$ is accomplished by a similarity transformation $U \hat{P} U^{+}$ applied to the standard $N$-dimensional representation of $S_{N}$, and dropping the first row and first column ( $\hat{P}$ is the regular $N$-dimensional representation).

Let us illustrate this approach on the problem of three spin polarized electrons $[13,14]$. For $N=3$ there is a CM particle (1) and two "relative particles" (2)(3). The anti-symmetrization of spin polarized states of relative harmonic oscillators leads to a severe restriction on possible fermionic states of the relative Hamiltonian

$$
\left|N_{2}, N_{3}, M_{2}, M_{3}\right\rangle^{\text {as }}=\frac{1}{\sqrt{2}}\left(\left|N_{2}, N_{3}, M_{2}, M_{3}\right\rangle-\left|N_{3}, N_{2}, M_{3}, M_{2}\right\rangle,\right.
$$

with the quantum number $L$ restricted to $L=M_{2}-M_{3}+N_{2}-N_{3}=3 m$, with $m=0,1,2,3 \ldots$ Hence statistics introduces additional symmetries which result in restricted coupled boson states. The Coulomb interaction conserves the total relative angular momentum $R=\left(M_{2}+M_{3}\right)-\left(N_{2}+N_{3}\right)$, which allows to diagonalize the relative Hamiltonian for each value of the relative angular momentum $R$. This can be done almost analytically when the time-reversal symmetry is broken. This "lowest Landau level" approximation is equivalent to setting 
$N_{2}=N_{3}=0$. A very simple sequence of the low angular momentum states $R=M_{2}+M_{3}$ can be constructed in analogy with Laughlin's approach [15]: $[\{|3,0\rangle\},\{|4,1\rangle\},\{|6,0\rangle\},\{|5,2\rangle\},\{|7,1\rangle\},\{|9,0\rangle,|6,3\rangle\} \ldots]$. The state with $R=4$ is missing and degeneracies proliferate only for $R>8$. The basic physics of this problem is best illustrated by considering the expectation value of the area spanned by 3 electrons: $\left\langle S^{2}\right\rangle=\left\{L^{2}+2+R\right\} / 4$. It is clear that for a given $R$ the area spanned by electrons is strongly maximized by having the largest possible $L$. The largest area for a series of "magic states" with $R=L=3 \mathrm{~m}$ minimizes any form of repulsive interaction energy, purely on the basis of many-particle symmetries. Which "magic state" is the ground state for a particular value of external parameters depends on the competition of potential $\sim 1 / R$ and kinetic $\sim R$ energy. An example of the extremely sparse energy spectrum of the relative Hamiltonian of a quantum dot is shown in Fig. 3a. For these parameters $\left(\omega_{N}=3.37 \mathrm{meV}, B=5 \mathrm{~T}\right.$,

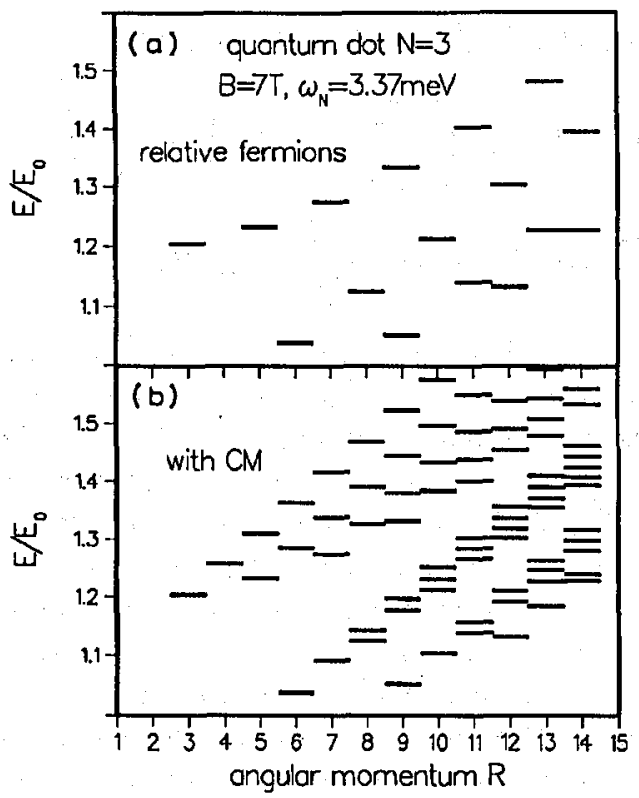

Fig. 3. (a) The energy spectrum of relative spin polarized electrons in the "lowest Landau level"; (b) the total energy spectrum, including CM excitations. Ground state at $R=6$ almost degenerate with state at $R=9$, quantum dot is close to a phase transition.

and other parameters applicable to GaAs) the ground state at the value of angular momentum $R=6$ is almost degenerate with the state at $R=9$, and the dot is almost compressible. For increasing value of the magnetic field higher angular momentum states $(R=9,12,15 \ldots)$ become the ground states. We now construct the total excitation spectrum $\left|M_{2}, N_{2}, M_{3}, N_{3}\right|^{\text {as }}\left|M_{1}\right\rangle$ by attaching the center of mass boson $\left(M_{1}\right)$ with energy $\Omega_{-} M_{1}$ to relative fermion states according to the total angular momentum $R_{\text {tot }}=M_{1}+R$ ( $R_{\text {tot }}$ remains a good quantum number $)$. The 
total spectrum, shown in Fig. 1b, is now completely equivalent to a spectrum one would obtain via direct diagonalization of the 3 -electron Hamiltonian in a basis of Slater determinants. Naturally, results of exact diagonalization yield states which appear to depend on the form of the electron-electron interactions. In our approach these states are simply constructed on the basis of symmetry. The number of states for each $R_{\text {tot }}$ in Fig. $3 \mathrm{~b}$ gives the total number of Slater determinants admixed into the highly correlated ground state. This degree of admixture is easily calculated from the overlap $\left\langle m_{1}, m_{2}, m_{3} \mid M_{2}, M_{3}\right\rangle$ of the relative fermion states with Slater determinants $\left|m_{1}, m_{2}, m_{3}\right\rangle$. To illustrate the charge distribution associated with

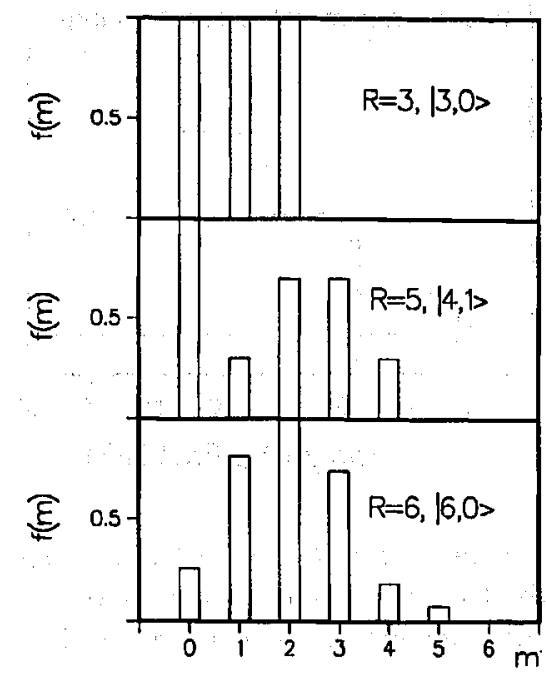

Fig. 4. Occupation $f(m)$ of single particle states in low angular momentum exact states $\left|M_{2}, 0\right\rangle$.

coherent states we show in Fig. 4 the occupations $f(m)$ of single particle states $|m\rangle$ $\left.\left.\left(f(m)=\sum_{m_{2}, m_{3}}\left|\left\langle m_{1}, m_{2}, m_{3} \mid R, L\right\rangle\right| M_{1}\right\rangle\right|^{2}\right)$ for a series of low angular momentum states. The charge distribution starts as a compact droplet at $R=3$ and proceeds to a charge distribution of a "ring" at $R=6$. Note that $R=3,6,9$ corresponds to a filling factor $\nu=1,1 / 2,1 / 3$ respectively. This has been interpreted [12] as an instability of the compact droplet against the formation of holes in the center of the droplet due to exchange interaction. We find that these states are completely independent of each other and are coherent states of relative fermions determined solely by many electron symmetries.

Without symmetry considerations, the size of the full Hilbert space, including spin and Landau level mixing, even for only 3 electrons is $\approx 10^{6}$. By using our symmetry analysis the problem becomes manageable. The calculated ground state energy as a function of the magnetic field is shown in Fig. 5, plotted with respect to the vacuum energy $E_{\mathrm{v}}=N\left(\Omega_{+}+\Omega_{-}\right) / 2$. Corresponding values of total spin $S$ and angular momentum $R$ are plotted below, including Zeeman energy of 


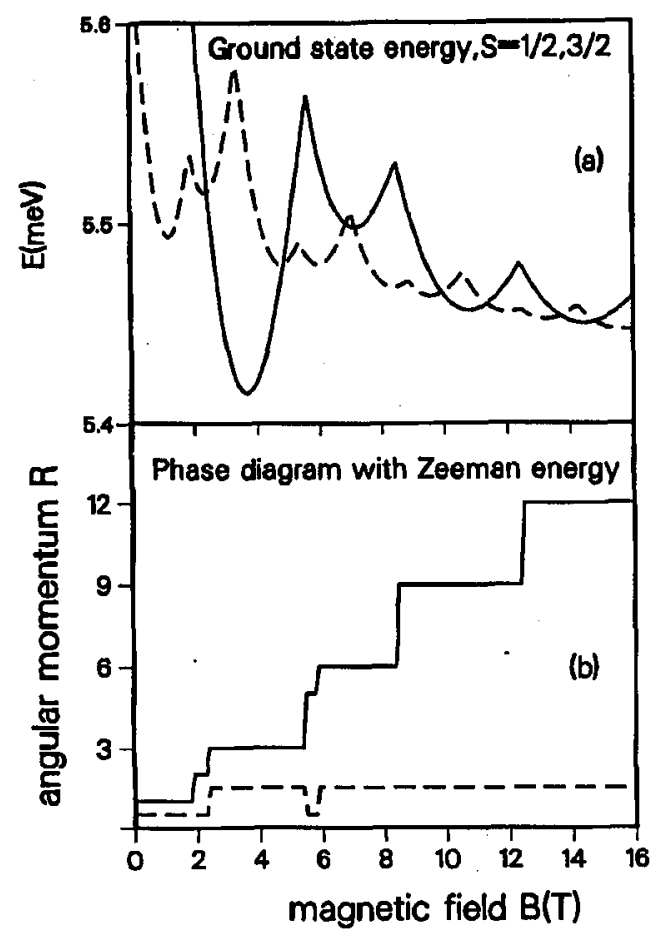

Fig. 5. (a) The ground state energy for $N=3$ electrons for spin polarized $(S=3 / 2$, solid line) and spin unpolarized ( $S=1 / 2$, dashed line) states without Zeeman energy, (b) the phase diagram (angular momentum, spin) of the dot including Zeeman energy.

$0.03 \mathrm{meV} / \mathrm{T}$. The cusps in the ground state energy as a function of the magnetic field are due to change in the spin and angular momentum polarization. At low $B$ electrons minimize their kinetic energy by occupying a small area of the dot, while as the magnetic field increases electrons maintain a larger area minimizing mutual repulsion. The area is quantized due to restrictions on possible quantum numbers $R$ and $L$ leading to incompressible spin polarized states with restricted values of relative angular momentum $R=3,6,9,12$.

These magic states have been observed by Ashoori et al. [17] via single electron capacitance spectroscopy. The effect of electron-electron interaction can be observed in radiative recombination spectra [14], transport [18], or in Raman scattering [19].

\section{References}

[1] For a review see J.J. Quinn, G. Eliasson, P. Hawrylak, Electromagnetic Waves: Recent Developments in Research, Vol. 1, Ed. P. Halevi, North-Holland, Amsterdam 1992.

[2] P. Hawrylak, NATO ASI 128, 295 (1993). 
[3] For recent reviews and references see M. Kastner, Phys. Today, p. 24, January 1993; T. Chakraborty, Comments Condens. Matter Phys. 16, 35 (1992).

[4] A. Pinczuk, G. Abstreiter, in: Light Scattering in Solids V, Eds. M. Cardona, G. Guntherodt, Springer, Berlin 1989, p. 153; P. Hawrylak, J.-W. Wu, J.J. Quinn, Phys. Rev. B 32, 5169 (1985).

[5] P. Hawrylak, Phys. Rev. Lett. 59, 485 (1987); P. Hawrylak, G. Eliasson, J.J. Quinn, Phys. Rev. B 37, 10187 (1988).

[6] P. Hawrylak, J.F. Young, P. Brockmann, Phys. Rev. B 49, 13624 (1994).

[7] C.S. Ting, T.K. Lee, J.J. Quinn, Phys. Rev. Lett. 34, 870 (1975); G.D. Mahan, B.E. Sernelius, Phys. Rev. Lett. 62, 2718 (1988); G.E. Santoro, G.F. Giuliani, Solid State Commun. 67, 681 (1988).

[8] G. Vignale, K.S. Singwi, Phys. Rev. B 32, 2156 (1985); Tai Kai Ng, K.S. Singwi, Phys. Rev. B 34, 7738 (1986); ibid., 7743 (1986).

[9] H.E. Rucker, E. Molinari, P. Lugli, Phys. Rev. B 45, 6747 (1992).

[10] Dai-Sik Kim, Jagdeep Shah, J.E. Cunningham, T.C. Damen, Stefan Schmitt-Rink, Wilfried Schaefer, Phys. Rev. Lett. 68, 2838 (1992).

[11] M.S. Skolnick, J.M. Rorison, K.J. Nash, D.J. Mowbray, P.R. Tapster, S.J. Bass, A.D. Pitt, Phys. Rev. Lett. 58, 2130 (1987); P. Hawrylak, Phys. Rev. B 44, 3821 (1991); M. Fritze, A. Nurmikko, P. Hawrylak, Phys. Rev. B 48, 4960 (1993).

[12] P. Maksym, Physica B 184, 385 (1993); P.A. Maksym, Tapash Chakraborty, Phys. Rev. Lett. 65, 108 (1990).

[13] P. Hawrylak, Phys. Rev. Lett. 71, 3347 (1993).

[14] P. Hawrylak, D. Pfannkuche, Phys. Rev. Lett. 70, 485 (1993); D. P fannkuche, Vidar Gudmundsson, P. Hawrylak, R.R. Gerhadts, Solid State Electron. 37, 1221 (1994).

[15] R.B. Laughlin, Phys. Rev. B 27, 3383 (1983); S.M. Girvin, Terrence Jach, Phys. Rev. B 28, 4506 (1983).

[16] Ch. Sikorski, U. Merkt, Phys. Rev. Lett. 62, 2164 (1989); B. Meurer, D. Heitman, K. Ploog, Phys. Rev. Lett. 68, 1371 (1992).

[17] R.C. Ashoori, H.L. Stormer, J.S. Weiner, L.N. Pfeiffer, K.W. Baldwin, K.W. West, Phys. Rev. Lett. 71, 613 (1993); Bo Su, V.J. Goldman, J.E. Cunningham, Science 255, 313 (1992).

[18] P.L. McEuen, E.B. Foxman, Jari Kinaret, U. Meirav, M.A. Kastner, N.S. Wingreen, S.J. Wind, Phys. Rev. B 45, 11419 (1992); A.S. Sachrajda, R.P. Taylor, C. Dharma-Wardana, P. Zawadzki, J.A. Adams, P.T. Coleridge, Phys. Rev. B 47, 6811 (1993).

[19] P. Hawrylak, to be published. 